Stoa

Vol. 11, no. 11, 2020, pp.138-166

ISSN 2007-1868

\title{
LOS TIPOS IDEALES DE WEBER Y LA IDEALIZACIÓN*
}

\author{
Juraj Halas
}

Los "tipos ideales" de Max Weber (TIS) han acaparado la atención de los filósofos de la ciencia. Proponentes de la Escuela de Poznan han formulado al menos dos diferentes reconstrucciones metodológicas de la concepción de Weber. Uno, debido a Izabella Nowakowa, ve los TIS como elementos extremos de clasificaciones y argumenta que el procedimiento empleado en la formación de éstos no es más que un caso especial del método de la idealización. Otro, debido a Leszek Nowak, explica los enunciados de tipos ideales como enunciados analíticos que desempeñan funciones heurísticas y explicativas. En este artículo evalúo estas reconstrucciones y las confronto con los textos originales de Weber. Muestro que ambos son inadecuados y propongo una tercera, basada en una concepción revisada de la abstracción y la idealización. También muestro que la función heurística de los TIS, como la discutiera Weber, consiste en su capacidad para formular explicaciones contrastantes.

\footnotetext{
* Este trabajo fue apoyado por la Agencia de Investigación y Desarrollo Eslovaca, bajo el acuerdo No. APVV-0149-12. Traducción de Adolfo García de la Sienra Guajardo y Saara Zulema Velázquez Sánchez. 


\section{Introducción}

Los escritos de Max Weber sobre los "tipos ideales" (TIS) han influido de manera importante en los debates sobre la filosofía y la metodología de las ciencias sociales y las humanidades. En el contexto de la escuela de filosofía de la ciencia de Poznan, han emergido al menos dos contribuciones para la reconstrucción de los TIS. A diferencia de la de Hempel, ambas enfatizan el papel de la idealización. El anterior enfoque histórico se debe a Leszek Nowak y fue formulado por primera vez en inglés en su (Nowak 1980). El segundo enfoque fue presentado por Izabella Nowakowa en (Nowakowa 2007). Este artículo examina críticamente ambas contribuciones. En la primera parte reformulo brevemente las explicaciones y argumento que ninguna de ellas captura adecuadamente la intención de la concepción de Weber. Por lo tanto, en la segunda parte presento una reconstrucción diferente, basada en parte en el entendimiento de Jones (2005) de los métodos de abstracción e idealización. Se muestra que la construcción de los TIS, tal y como los describe Weber, involucra la aplicación de ambos métodos. Propongo que se vean los TIS como objetos ideales similares en características al "punto de masa" o al "péndulo simple" de la física. Al analizar uno de los ejemplos de Weber del uso de los TIS, concluyo que su relevancia heurística reside en su papel en la formulación de explicaciones contrastantes del fenómeno social.

\section{La escuela de Poznan sobre los TIS de Weber}

Antes de pasar a las reconstrucciones en cuestión, parece apropiado resumir brevemente algunos de los puntos clave de la concepción de Weber, a fin de llegar a un estándar con el que se puedan comparar los análisis metodológicos.

En la concepción de Weber, todas las ciencias aspiran a un "ordenamiento intelectual de la realidad empírica" (Weber 2012c, p. 102) por medio de "conceptos" (Begriffe). Debido a que los fenómenos se caracterizan por una "multiplicidad infinita", tanto "extensiva" como "intensiva" (Weber 2012e, p. 40), su procesamiento en conceptos es necesariamente selectivo. Para Weber, todos los conceptos son el resultado de un proceso de abstracción que separa aquellos aspectos de la realidad considerados relevantes (con respecto a ciertos objetivos cognitivos) de aquellos que no lo son. En línea con la tradición neokantiana, Weber distingue dos tipos principales de abstracción: la generalización, la cual se enfoca en las características comunes compartidas por diversos fenómenos y los conjunta en un solo concepto, y la individualización 
o aislamiento, la cual extrae los aspectos específicos de un fenómeno particular y desconoce aquellos que presentan algo en común con otros fenómenos. Estas dos formas de "formación de conceptos" (Begriffsbildung) se hallan en la base de dos estrategias cognitivas diferentes y de dos tipos diferentes de ciencias.

El objetivo de las ciencias naturales es el ordenamiento de la realidad por medio de "conceptos relacionales" o "leyes" que contienen la forma de "ecuaciones causales" (Weber 2012e, p. 5). Formados por la abstracción generalizadora, tales conceptos generales permiten la subsunción de los fenómenos más dispares. El precio a pagar por la extensión relativamente amplia de tales conceptos es su contenido relativamente magro: representan la realidad empírica como carente de cualidades (Weber 2012e, p. 5). Sin embargo, con respecto a los objetivos cognitivos de las ciencias naturales, esto no presenta un problema. ${ }^{2}$ En contraste, la tarea de las ciencias sociales consiste en "adquirir conocimiento de la realidad, con su carácter constante y universal de diferenciación cualitativa y singularidad" (Weber 2012e, p. 5); "lo que nos importa en las ciencias sociales es el aspecto cualitativo de los eventos" (Weber 2012c, p. 115). Correspondiendo a esta meta está la abstracción individualizadora como un modo de formación de conceptos. Sin embargo, ante la infinita multiplicidad de todos los fenómenos, se requiere un criterio adicional para seleccionar aquellos aspectos de un evento que no sólo son exclusivos de él, sino que también se consideran más relevantes que otros aspectos similares. Este criterio es la "relación de valor" (Wertbeziehung): ${ }^{3}$ en sus conceptos, las disciplinas científicas sociales captan aquellos aspectos de los fenómenos que son relevantes con respecto a ciertos valores. ${ }^{4}$

\footnotetext{
${ }^{2}$ En este respecto, la concepción de Weber se alimenta de la doctrina tradicional, ahora fuera de moda, de la proporcionalidad inversa entre la extensión y el contenido (intensión, comprehensión) de un concepto. Sin embargo, No trataré en más detalle este aspecto de la concepción de Weber.

${ }^{3}$ Las traducciones primeras al inglés de los escritos de Weber usan el apropiado término (valuerelevance) "relevancia de valor".

${ }^{4}$ Para los propósitos de este artículo, será suficiente el siguiente ejemplo: al formar el concepto de un evento histórico único (como la I Guerra Mundial), el historiador puede elegir de entre un número infinito de aspectos que lo distinguen de otros eventos. Basado en el criterio de la relación de valor, elige aquellos que son relevantes con respecto a los problemas culturales que él (o "su tiempo") considera importantes. Así, el aspecto de la I Guerra Mundial que amerita la inclusión en el concepto correspondiente podría, dependiendo del contexto más amplio, ser el imperialismo, el uso carente de precedentes históricos de los tanques, o el hecho de que la guerra estalló poco después del asesinato del Archiduque Francisco Fernando, etcétera. Y debido a que el contexto de valores que forma el trasfondo de estas disciplinas está constantemente cambiando, es legítimo que los investigadores regresen a los fenómenos que ya habían sido tratados por otros investigadores desde el punto de vista de valores
} 
Se debe señalar que Weber nunca vio la distinción entre ciencias naturales y sociales como absoluta - en el sentido de que las segundas nunca habrían de usar la abstracción generalizadora y las primeras nunca habrían de formar conceptos individuales mediante la abstracción aisladora. Incluso una disciplina científica natural puede adoptar un punto de vista histórico; es decir, individualizador, como cuando la geografía física, por ejemplo, estudia el origen de una montaña particular. De manera similar, una disciplina científica social puede formar generalizaciones nómicas que capturan característias comunes de muchos fenómenos dispares. De acuerdo con Weber, la distinción sólo se aplica estrictamente a la "mecánica pura" y a "ciertas partes de la ciencia histórica" (Weber 2012e, p. 6); en el caso de las otras disciplinas, solo se refiere a las metas cognitivas predominantes y a la práctica metodológica.

Además de los conceptos que son el resultado de uno de los dos tipos de abstracción discutidos previamente, Weber identifica otro tipo de conceptos, supuestamente específicos de las disciplinas científicas sociales. Como ejemplos de este tercer tipo, él enlista el "intercambio económico", el "capitalismo", el "cristianismo" (Weber 2012c, p. 31) o el "sujeto económico" (en el sentido de un "homo oeconomicus") (Weber 1990, p. 30). Otros ejemplos de este tipo son conceptos formados por el propio Weber: "competencia", "organización", "institución” y muchos otros en (Weber 1968). Por un lado, estos conceptos parecen generales, porque aparentemente se pueden incluir muchas ejemplificaciones bajo ellos — casos particulares de intercambio económico, formas históricas propias del capitalismo, etcétera (Burger 1987, p. 122). Por otro lado, ninguno de los ejemplos particulares satisface completamente los criterios postulados por el concepto: en cada caso de intercambio de mercado, las circunstancias y los motivos de los agentes involucrados se distinguen del intercambio "puro" postulado por la teoría económica (el cual ocurre, por ejemplo, cuando todos los agentes son perfectos maximizadores de la utilidad). Desde el punto de vista de la teoría de los conceptos que Weber suscribió, éstos no pueden ser conceptos generales del tipo habitual, pero tampoco son conceptos individuales. Weber los denomina "tipos ideales".

De modo similar a otros tipos de conceptos, los TIS sirven para "ordenar la realidad empírica". También se forman por abstracción, si bien una que es específica. Implica una "acentuación teórica de ciertos elementos de la realidad" (Weber 2012c: 124), una “acentuación unilateral de uno o varios puntos 
de vista" y la "síntesis de muchos fenómenos individuales difusos y discretos (más presentes en un lugar, menos en otros y ocasionalmente completamente ausentes)" en "una imagen mental internamente consistente" (Weber 2012c, p. 125). Tales conceptos no tienen ejemplificaciones particulares en la realidad empírica; son "no realistas" (Weber 1968, p. 21) y sólo "se aproximan más o menos [a la realidad]" (Weber 2012b, p. 331).

Como ya se mencionó, Weber pensó que los TIS sólo ocurren en las ciencias sociales. Esto se debe a la complejidad de su objeto de estudio, ${ }^{5}$ la cual es en última instancia consecuencia de la intencionalidad humana (Burger 1987: 116). Un cierto tipo de acción humana — digamos, el intercambio económico- siempre está motivado por un conjunto de razones, metas y valores heterogéneos. Por lo tanto, la abstracción generalizada es incapaz de formar un concepto general del mismo que abarque todos los casos. La abstracción individualizada, por otro lado, no puede abarcar más de un caso parTiular simple. Un tipo ideal debería permitirle a uno entender qué forma adoptaría la acción si estuviese motivada exclusivamente por razones particulares explícitamente formuladas. Cuáles de éstas serán el foco de un TI parTiular está determinado - al igual que en el caso de la abstracción individualizadorapor el criterio de la relación de valor. ${ }^{6}$ Para Weber, una característia específica del TI como "concepto" es, por lo tanto, no sólo que no es realista, sino que también se interesa por la acción humana y su "significado" — en el sentido de los motivos atribuidos a la acción por el agente en acción o por otros. Weber se dio cuenta de que las ciencias naturales usaban conceptos como "masa puntual" o "espacio absolutamente vacío" y obviamente los veía como términos análogos en algún sentido a los TIS, ${ }^{7}$ pero sólo hablé de los TIS con respecto a la "acción significativa"; es decir, en el contexto de las ciencias sociales.

Él vio la función de estos conceptos como doble: expositiva y heurística. ${ }^{8}$ En cuanto a la primera, los TIS permiten formular descripciones y clasificaciones inequívocas de fenómenos sociales, si bien a costa de la "abstracción"

5 "Por ejemplo, el mismo fenómeno histórico puede ser feudal en un aspecto, en otro patrimonial, en otro burocrátio, y todavía en otro carismátio. Para darle un significado preciso a estos términos, es necesario que el sociólogo formule tipos ideales puros de las correspondientes formas de acción [...]" (Weber 1968, p. 20).

6 “Así como hay por lo tanto diferentes 'puntos de vista' desde los cuales podemos considerar estos fenómenos como importantes para nosotros, así puede uno basarse en principios enteramente diferentes para la selección de aquellas relaciones que han de ser incluidas en el tipo ideal [... ]" (Weber 2012c, p. 125).

7 Véase, por ejemplo, (Weber 1990, p. 30), (Weber 1968, p. 20).

${ }^{8}$ Véase, por ejemplo, Weber 2012e, p. 74), (Weber 2012c: 125), (Weber 1968, p. 21). 
(Weber 1968: 22). En otras palabras, un TI "no es una representación de la realidad, sino que busca proporcionar la explicación [científica] con medios de expresión no ambiguos" (Weber 2012c: 125). Con respecto a esto último, un TI sirve para "comparar" la realidad empírica con él, para establecer cómo contrasta con la realidad, cuán lejos o relativamente cerca se encuentra de la realidad" (Weber 2012b, p. 331), así como para "dirigir la formulación de hipótesis" (Weber 2012c, p. 125) la cual explicaría porqué la acción humana observada difiere de una típica ideal. Los TIS funcionan como un instrumento clave en la formulación de explicaciones causales en las ciencias sociales: "para aprehender las interconexiones causales reales, construimos unas irreales" (Weber 2012a, p. 182).

Regresaré en la Sección 2 a considerar detalles adicionales de la "construcción" de los TIS y su uso como instrumentos heurísticos. El material cubierto hasta ahora será suficiente para evaluar la adecuación de las dos reconstrucciones de la concepción weberiana, a lo cual paso ahora.

\subsection{Nowakowa: elemento extremo de una clasificación}

Izabella Nowakowa (2007) se propuso comparar la concepción de los TIS de Weber con la idealización hegeliana tal y como está formalizada en la filosofía de la ciencia de la idealización. Un TI, argumenta, puede ser visto como un "objeto posible" resultante de una "deformación" específica de un objeto real —en otras palabras, de una atribución contrafáctica de un valor mínimo de una cierta propiedad (o propiedades) del objeto real (Nowakowa 2007, p. 164). Siguiendo las anteriores clasificaciones de procedimientos de deformación, ella llama a esta operación "potencialización negativa". Dado que Nowakowa ve la idealización, en línea con la tradición de Poznan, como una combinación de dos tipos de procedimientos de deformación (la "reducción", es decir la eliminación de propiedades, y la "potencialización negativa"), Nowakowa concluye que el procedimiento involucra la construcción de los TIS weberianos como un caso especial de idealización simple.

En la explicación de Nowakowa, un TI es el miembro extremo de una clasificación. En una escala ascendente de objetos basada en una propiedad dada, el elemento $S_{0}$ es un TI si está vacío, es decir, si no hay objetos reales que satisfagan el valor (mínimo) dado de la propiedad (Nowakowa 2007, p. 160). Por el contrario, los elementos $S_{1}, \ldots, S_{n}$ de la clasificación son "tipos reales" que son ejemplificados mediante objetos reales. Así, el papel de los 
TIS, de acuerdo con Nowakowa, parece ser sistematizador: los TIS sirven como estándar con el cual los tipos reales se comparan.

Antes de pasar a una crítica de esta explicación, cabe señalar que Nowakowa reconoce el "carácter puramente conceptual" de sus conclusiones, así como el hecho de que la explicación se basa en reconstrucciones anteriores, y no en material original de Weber (Nowakowa 2007: 159 y 164). Aun así, existe evidencia en Weber a favor de su enfoque: por ejemplo, Weber caracteriza los TIS como un "concepto límite contra el cual la realidad es medida — con el cual es comparada" (Weber 2012c, p. 127). Sin embargo, parece haber dos razones por las cuales la explicación de Nowakowa es deficiente.

Primeramente, esta idea no aborda la cuestión de la función heurística de los TIS. Los intentos por explicar el TI de este modo, es decir como un "concepto limite" en el sentido de elemento extremo de una clasificación, tiene una larga historia. Sin embargo, como fue señalado por Hans Albert:

Si examinamos el uso de los tipos ideales en Max Weber, parece no estar en sintonía con el análisis de (los TIS como un elemento extremo) (...) A través de las construcciones típico ideales, Weber evidentemente deseaba proveer una fundamentación para la explicación de la realidad social. (Albert 1967, p. 57) ${ }^{9}$

Nowakowa justifica su enfoque al referirse a la "reconstrucción propuesta por C. G. Hempel y P. Oppenheim como una explicación de la tradición weberiana" (Nowakowa 2007, p. 159). Claramente, la referencia implícita aquí es a la innovación (Hempel y Oppenheim 1936). Pero ni ese trabajo, ni su posterior desarrollo en (Hempel 1960), aborda los tipos ideales. El objeto de estudio de estas contribuciones son las tipologías y los tipos como se encuentran, por ejemplo, en las teorías psicológicas de la personalidad, donde desde luego desempeñan un papel sistematizador. De hecho, Hempel analizó los TIS de Weber y su papel explicativo (1965), pero no los explicó como conceptos comparativos, sino como teorías.

En segundo lugar, parece que Nowakowa se equivoca al reducir el procedimiento de deformación utilizado para construir tipos ideales a la "potencialización negativa". En un manuscrito inédito de conferencias sobre economía, Weber analiza el método mediante el cual la teoría económica introduce el “objeto económico interpretado". La economía:

[... ] (a) trata como ausentes —ignora todos los motivos que influyen en el Hombre empírico que no son específicamente económicos; es decir, que no se originan

${ }^{9}$ Para conclusiones similares, véase (Janoska-Bendl 1965, p. 78) y (Burger 1987, p. 158). 
en la satisfacción de las necesidades materiales; (b) finge la existencia de ciertas cualidades que el Hombre empírico no tiene o presenta de manera imperfecta [...]. Los argumentos de la economía se relacionan con un hombre irrealista, análogo a la figura ideal en las matemáticas” (Weber 1990, p. 30)

Dado que Weber vio a la economía como un caso paradigmático de una disciplina que usa los TIS, $^{10}$ es seguro suponer que lo anterior describe la construcción de un TI. Al confrontarlo con la reconstrucción de Nowakowa, uno puede objetar que no tiene en cuenta la diferencia entre el "ignorar" (es decir, la eliminación o reducción de una propiedad) y la atribución explícita de un valor mínimo (es decir, la potencialización negativa). Además, ignora completamente el caso (b), es decir, la atribución de propiedades que el objeto original (en este caso, el "Hombre empírico") no tiene. En el vocabulario de Poznan, esto se denominaría "trascendentalización". La conclusión de Nowakowa de que el procedimiento de Weber es simplemente un caso especial de idealización - en la medida en que éste se entiende como una combinación de reducción y potencialización negativa- es por lo tanto falsa.

\subsection{Nowak: enuncación analítica}

En su relato clásico de la filosofía idealizadora de la ciencia, Leszek Nowak (1980) también analiza los TIS de Weber. Él propone verlos como enunciados de la forma "Si $A_{1}(x) \wedge \cdots \wedge A_{m}(x)$ entonces $B(x)$ ". Debido a que $B$ se refiere a una propiedad que es satisfecha por definición por cualquier objeto con las propiedades $A_{1}, \ldots, A_{m}$ tales enunciados típicos ideales son analíticos. Las propiedades involucradas pueden o no ser ejemplificadas por algún objeto real (Nowak 1980, pp. 48-49).

El papel desempeñado por los Tis en la explicación depende, según Nowak, de si "se desvían de la realidad" o no (Nowak 1980: 49). Esta pregunta puede ser contestada sometiendo a prueba la hipótesis acerca de la aplicabilidad de la declaración típica ideal a una hipótesis dada; es decir, un enunciado de la forma $A_{1}(a) \wedge \cdots \wedge A_{m}(a)$. Si la prueba es positiva, la explicación puede ser formulada con base en el siguiente esquema (Nowak 1980: 49):

10 "La teoría económica se revela como una suma de conceptos 'típicos ideales'” (Weber 2012g, p. 249). 


$$
\begin{aligned}
& (\forall x)\left(A_{1}(x) \wedge \cdots \wedge A_{m}(x)\right) \rightarrow B(x) \\
& A_{1}(a) \wedge \cdots \wedge A_{m}(a) \\
& \hline B(a)
\end{aligned}
$$

El hecho de que $a$ tiene la propiedad $B$ se explica refiriéndose al hecho que tiene las propiedades $A_{1}, \ldots A_{n} \mathrm{y}$ al enunciado TI.

En caso de que el objeto investigado no posea la propiedad B, la declaración del TI cumple una función heurística. Debe guiar al investigador a buscar una propiedad $C$ que impida que el objeto tenga una de las propiedades $A_{1}, \ldots, A_{m}$ (y por lo tanto también $B$ ). La explicación contiene la siguiente forma (Nowak, 1980, 50):

$$
\begin{aligned}
& (\forall x)\left(C(x) \rightarrow \neg A_{1}(x)\right) \\
& C(a) \\
& \neg A_{1}(a)
\end{aligned}
$$

Una característica importante de la reconstrucción de Nowak es que enfatiza la relación entre los TIS y la explicación, y considera su función heurística. Sin embargo, la solución de Nowak presenta problemas. Para hacerlos explícitos, es necesaria una mirada más cercana a algunos de los detalles.

Dado que Nowak no proporciona una ilustración de la forma en que los TIS funcionan en la práctica, construiré una a partir del siguiente ejemplo weberiano, al que Nowak también hace referencia:

Uno puede, por ejemplo, llegar a la conclusión teórica de que en una sociedad que está organizada sobre principios "artesanales" estrictos, la única fuente de acumulación de capital sólo puede ser la renta del suelo. Quizá a partir de esto uno pueda - pues la corrección del constructo no se cuestiona aquí- construir una imagen ideal pura del cambio, condicionado por ciertos factores específicos, — por ejemplo, tierra limitada, el aumento de la población [...] - de una economía artesanal a una organización económica capitalista. Si el curso empírico-histórico del desarrollo fue realmente idéntico al construido, sólo puede investigarse mediante el uso de un dispositivo heurístico para la comparación del tipo ideal y los "hechos". Si el tipo ideal se construyera "correctamente" y el curso real de los acontecimientos no correspondiera al predicho por el tipo ideal, se demostraría la hipótesis de que la sociedad medieval no era, en ciertos aspectos, un tipo de sociedad estrictamente 
"artesanal". Y si el tipo ideal fuera construido de una manera heurística 'ideal' sí, y de qué manera, podría ocurrir esto en nuestro ejemplo será aquí ignorado por completo- guiará la investigación hacia un camino conducente a un entendimiento más preciso de los componentes no artesanales de la sociedad medieval en sus características particulares y su importancia histórica. Si conduce a este resultado, cumple su propósito lógico, aunque, al hacerlo, demuestre su divergencia con la realidad" (citado en Nowak 1980, p. 48, omisiones de J. H.)

En este caso, parece que el antecedente del enunciado de TI se refiere a las propiedades "estar organizado sobre principios artesanales estrictos" $\left(A_{1}\right)$, "tener tierra limitada" $\left(A_{2}\right)$, "tener una población creciente" $\left(A_{3}\right)$, mientras que el consecuente se refiere a la propiedad "cambia hacia una organización económica capitalista" $(B)$. Investigando el surgimiento de una economía capitalista en una sociedad dada, el investigador confrontaría datos sobre su forma anterior de organización económica con el enunciado de TI. Si la pareciera que las sociedad satisface el antecedente podría — suponiendo que el TI esté "correctamente construido"- explicar el surgimiento de una economía capitalista como resultado de las propiedades $A_{1}, \ldots A_{3}$ Sin embargo, Weber no considera este caso como ejemplo y supone que el curso real de los eventos no se correspondería con el típico ideal. Esta discrepancia significa, en los términos de Nowak, que la sociedad estudiada no tiene la propiedad $B$ (es decir, que difiere en ciertos aspectos de la imagen construida de una economía capitalista), y por lo tanto también carecía de al menos una de los propiedades $A_{1}, \ldots A_{3}$. Si se supone que la presencia de las propiedades $A_{2}$ y $A_{3}$ pueden confirmarse de manera confiable e independiente, uno puede inferir que la sociedad carecía de la propiedad $A_{1}$; es decir, no era una sociedad organizada en estrictos principios artesanales. Una investigación adicional intentará identificar las circunstancias específicas $(C)$ debido a las cuales la economía de la sociedad difiere de la de una economía puramente artesanal y, en última instancia, formularía una explicación basada en el segundo esquema de Nowak.

Pese a esto, no está claro por qué Nowak considera que las declaraciones de los TIS son analíticas. Apegándose al ejemplo citado y reconstruyendo el enunciado como "Si la economía de cierta sociedad está organizada en estrictos principios artesanales y esta sociedad tiene tierra limitada y ..., entonces la economía de esta sociedad se transformará en capitalista", no parece que estemos tratando con un enunciado analítico ni siquiera relativo a la "conclusión 
teórica" con la que Weber introduce el ejemplo. Supongamos, sin embargo, que los enunciados de TI fueran realmente analíticos. Al suponer la definición operativa estándar de la propiedad "ser un ácido", el enunciado "En todos los líquidos $\left(A_{1}\right)$ que son ácidos $\left(A_{2}\right)$, un papel tornasol se volverá rojo $(B)$ " es analítico y calificaría como un enunciado de TI de acuerdo con el criterio de Nowak. El hecho de que después de sumergir un trozo de papel tornasol en el líquido $b$, el papel se puso rojo, podría entonces ser explicado con referencia a que $b$ es un ácido y al enunciado de TI. Por el contrario, si en algún líquido el papel tornasol no se vuelve rojo, se puede inferir que no es un ácido. Entonces se puede intentar confirmar el propiedad $C$ ("ser una muestra de agua de la llave") incompatible con $A_{2}$. Finalmente, uno puede formular la explicación utilizando el segundo esquema proporcionado por Nowak:

El agua de la llave no es un ácido

$b$ es una muestra de agua de la llave

b no es un ácido

El objetivo de este ejercicio es mostrar que, si los enunciados de TI fueran analíticos, su importancia para la ciencia empírica, cuyo objetivo es la explicación causal, en el mejor de los casos sería limitada. En el primer caso, la "explicación" es puramente explicativa. En el segundo caso, no solo el enunciado de TI no aparece en la explicación (como reconoce Nowak), sino que también su papel heurístico es cuestionable. Si es cuestión de definición que cualquier objeto con las propiedades $A_{1}$ y $A_{2}$ tiene la propiedad $\mathrm{B}$, entonces la inferencia de la ausencia de $B$ a la ausencia de $A_{1}$ o $A_{2}$ difícilmente puede ser visto como un descubrimiento significativo. Más aún, Nowak no plantea la pregunta de cómo el enunciado de TI podría ayudar en la identificación de propiedades (por ejemplo, $C$ ), debido a que el objeto real diverge del TI.

Sin embargo, los problemas no terminan aquí. Nowak parece justificar el análisis de los enunciados de TI por el hecho de que "no se pueden someter a prueba en lo absoluto" (Nowak 1980, p. 48). Por otro lado, concede la posibilidad de que el enunciado TI "concuerde con el fenómeno investigado" o "no corresponda al fenómeno real" (Nowak 1980, p. 48). No está claro cómo es posible pronunciar que un enunciado analítico, carente de contenido empírico, corresponde o no a los hechos. Si un enunciado no puede ser sometido a prueba en lo absoluto, uno ni siquiera debería ser capaz de plantear la cuestión de tal correspondencia. 
Dificultades adicionales de la reconstrucción de Nowak tienen que ver con el concepto de TI. Nowak no especifica qué tipo de objeto es un TI. En cambio, prefiere los términos "concepto típico ideal" y "enunciado típico ideal". El primero es un concepto que "denota" un TI (Nowak 1980, p. 41), el segundo una declaración que "se refiere" a un TI (Nowak 1980, p. 48). Con el fin de explicar el concepto de TI, Nowak cita un breve pasaje del "Objetividad" de Weber, ${ }^{11}$ que infortunadamente, no aborda la cuestión de qué es un TI. Por lo tanto, la naturaleza de los procedimientos utilizados en la construcción de los TIS no es revelado.

En un texto posterior, Nowak parece haber revisado su reconstrucción original, en la misma dirección que la sugerida por Nowakowa (2007):

El paradigma neoweberiano. La idealización es básicamente un método de construcción de nociones científicas. Teniendo una cierta tipología en mente, uno puede identificar su miembro extremo. Si el miembro es un conjunto vacío, se denomina un tipo ideal y la noción unida a él se etiqueta como idealización [... ]. La fuente de este enfoque radica en la metodología de Max Weber. En la filosofía moderna de la ciencia es la concepción de Hempel la que es una explicación de las ideas weberianas [...]" (Nowak 2000, p. 1)

Aquí, Nowak se refiere a Hempel —Oppenheim (1936) y Hempel (1960)— quienes, como se mencionó anteriormente, no se ocupan de los TIS de Weber en lo absoluto.

Para resumir, ninguna de las sugerencias discutidas proporciona una descripción adecuada de los métodos utilizados en la construcción de los TIS, o una reconstrucción adecuada de sus usos para explicar los fenómenos sociales, que esté libre de problemas conceptuales. En la siguiente sección, se propone una relatoría de la concepción de Weber de que abarca ambos aspectos.

\footnotetext{
11 "Un tipo ideal es formado por la acentuación unilateral de uno o más puntos de vista y por la síntesis de muchos fenómenos individuales concretos difusos, discretos, más o menos presentes y ocasionalmente ausentes, que son ordenados de acuerdo con aquellos puntos de vista unilateralmente enfatizados en un constructo analítico unificado. En su pureza conceptual, este constructo mental no se puede encontrar empíricamente en ninguna parte en la realidad. Es una Utopía". (Citado en Nowak 1980: 41). Debe notarse que la traducción inglesa anterior citada por Nowak tiene "constructo analítico" en lugar del original Gedankenbild, el cual prima facie favorece la interpretación de Nowak de los TIS como enunciados analíticos. En la traducción más reciente se utiliza el más adecuado "imagen mental" (Nowak 2012c, p. 160).
} 


\section{Abstracción, idealización y TIS}

En esta sección primero muestro que los TIS pueden ser vistos como objetos abstractos (es decir, no espaciotemporales), los cuales resultan de la aplicación de métodos de abstracción e idealización. Estos son métodos no empíricos que son continuamente usados en las ciencias naturales y en las sociales. Por lo tanto, argumento que los procedimientos empleados en la construcción de TIS no son en principio diferentes de los utilizados en las ciencias naturales cuando introducen objetos ideales como el "péndulo simple". Luego me muevo hacia la reconstrucción del método involucrado en el uso heurístico de los TIS en la explicación causal de la acción humana. El llamado método del tipo ideal es así un complejo meta-método que consta de dos métodos: la construcción del TI y su uso heurístico. ${ }^{12} \mathrm{Al}$ reconstruir ambos métodos, tomo elementos de un modelo del método como una secuencia de instrucciones, así como de una explicación de los métodos de abstracción e idealización en ese modelo. $^{13}$

\subsection{La "construcción" de los TIS}

En la Sección 1 hemos visto a Weber describir el proceso de construcción de los TIS como una "acentuación teórica" en la cual las propiedades de los objetos reales (espaciotemporales) se tratan "como ausentes", mientras se pretende la existencia de otras propiedades. En otra parte, Weber caracteriza este procedimiento como un "proceso de abstracción" cuyo resultado es una "imagen imaginaria" o un "constructo teórico" (Weber 2012a, p. 175). Weber también enfatizó que un TI es "unido a partir de las partes individuales tomadas de la realidad histórica" (Weber 1992, p. 13), es decir, que la fuente de su material es aquello que está "inmediatamente dado" (Weber 2012a,175). A partir de esto, dos conclusiones relevantes para la explicación del método de los TIS se pueden extraer. Primero, si un método se entiende como una secuencia de instrucciones que guían a transformar un determinado objeto de entrada a uno de salida, entonces la construcción de los TIS consiste en la transformación de una cierta representación verídica de la "realidad histórica" (por ejemplo, una representación del "Hombre empírico") mediante una "imagen imaginaria" (por ejemplo, el "sujeto económico") que distorsiona, en cierto sentido, la representación verídica original.

\footnotetext{
${ }^{12}$ Hago abstracción a partir de la función expositiva de los TIS, ya que tampoco había sido el foco de los escritos de Weber.

${ }^{13}$ Véase Bielik et al. (2014a, b, c, d) y Halas (2015).
} 
En segundo lugar, dos tipos de operaciones distorsionadoras están involucradas en esta transformación: el ignorar algunas de las propiedades del objeto input y la especificación contrafactual de las "nuevas" propiedades que no son ejemplificadas por el objeto input. Siguiendo a (Jones 2005), llamo estas operaciones "abstracción" e "idealización”, respectivamente. En (Halas 2005) he propuesto la explicación de estos métodos en términos de secuencias de instrucciones. Ambos métodos toman un objeto abstracto (por ejemplo, un objeto que no es espacio temporal) como su input y producen un objeto abstracto diferente como output. El objeto input puede o puede no ser una representación (verídica de algún objeto espaciotemporal; pero también puede ser producto de previas abstracciones y/o idealizaciones. Doy por sentado que el método de abstracción consiste en los siguientes pasos:

(1) Identificación del objeto input $o$.

(2) Identificación del conjunto de propiedades $A$ codificadas ${ }^{14}$ por $o$ que son relevantes con respecto a los objetivos cognitivos presentes.

(3) Definición del objeto output $o^{\prime}$ que codifica propiedades del conjunto A.

(4) ¡Declaración de que $o^{\prime}$ es el abstractum basado en $o$.

En contraste, el método de idealización consiste en las siguientes instrucciones:

(1) Identificación del objeto input $o$.

(2) Identificación del conjunto de propiedades $A$ codificadas por $o$.

(3) Identificación del conjunto de propiedades $A_{n} \subset A$ codificadas por $o$ que no se conforman a los objetivos cognitivos presentes.

(4) Identificación del conjunto de propiedades $A_{i}$ que se conforman a los objetivos cognitivos presentes.

(5) Definición del objeto output $o^{\prime}$ que codifica las propiedades del conjunto $A^{\prime}=\left(A \backslash A_{n}\right) \cup A_{i}$.

(6) Declaración de que $o^{\prime}$ es el objeto ideal basado en $o$.

${ }^{14}$ Sobre la distinción entre la ejemplificación y la codificiación de propiedades, con respecto a objetos abstractos, véase (Zalta 1988, pp. 15 ss.). 
Por ende, un abstractum no codifica algunas de las propiedades codificadas por el objeto input, mientras que un objeto ideal codifica propiedades que no estaban codificadas por el objeto input. En ambos casos, la selección de las propiedades codificadas por el objeto output está gobernada por los objetivos cognitivos más amplios en prosecución de los cuales el objeto input está siendo transformado.

Para ilustrar el entendimiento propuesto de ambos métodos, consideremos un objeto típico ideal de la física: el "punto de masa". Su introducción fue motivada por ciertas finalidades teóricas y tiene lugar en el trasfondo de conocimiento preexistente. Puede ser construido como una transformación de un objeto input, por ejemplo una representación de un cuerpo físico genérico (él mismo un objeto abstracto, si bien uno que está ejemplificado por objetos particulares espaciotemporales), en un objeto output. La transformación procede en dos pasos secuenciales. En el primer paso, todas las propiedades excepto la de "tener masa", "tener volumen (no cero)" y "tener posición" son abstraídas. En el segundo paso, la propiedad "tener volumen distinto de cero" es reemplazada con "tener volumen cero". El objeto ideal resultante solamente codifica las propiedades "tener masa", "tener volumen cero" y "tener posición".

\section{Objetivos cognitivos: tres criterios}

La construcción de un TI puede ser comprendida como un procedimiento que combina abstracción e idealización y que está guiada por objetivos cognitivos específicos. Las especificidades de estos objetivos — qué propiedades particulares habrán de ser abstraídas, que propiedades serán idealizadas- diferirán de un caso a otro. Sin embargo, es posible identificar en la concepción de Weber ciertos lineamientos generales que vió como imperativos. De acuerdo con Weber, cada TI debe satisfacer los tres criterios:

(1) el criterio de la "relación de valor",

(2) el criterio de la "adecuación sobre el nivel de significado" ("adecuación del significado"),

(3) el criterio de la "adecuación causal".

El primer criterio de Weber ya ha sido mencionado; en su concepción, se aplica universalmente a la formación de conceptos en las ciencias sociales. 
Con respecto a la abstracción y a la idealización involucradas en la construcción de un TI, este criterio conduce a la selección de propiedades (aquellas que, en el caso de las abstracción, no son ignoradas, y, en el caso de la idealización, son contrafactualmente atribuidas al objeto resultante) que son "relevantes en valor". Sin embargo, de acuerdo con Weber, no hay una prueba singular y conclusiva de relevancia de valor. Como hemos visto, Weber permite la existencia de diferentes TIS construidos a partir del mismo material original, pero sobre el trasfondo de diferentes puntos de vista de valor. Por lo tanto algunos críticos han acusado a Weber de "voluntarismo", "subjetivismo" o "decisionismo". ${ }^{15}$ pero, aunque Weber permitió algún grado de libertad en la construcción de los TIS, esto no fue el caso para su uso heurístico. Solamente algunas de las TIS que pueden ser construidas sobre la base de diferentes valores resultarán ser útiles:

$\mathrm{y}$, desde luego, nunca es posible determinar por adelantado si [tales esfuerzos constructivos] son meras fantasías o si constituyen una formación de conceptos científicamente fructífera. Aquí, también, el único estándar es si [el tipo ideal] es útil para adquirir conocimiento de fenómenos culturales concretos - su contexto, su determinación causal y su importancia. Consecuentemente, la construcción del tipos ideales abstractos sólo puede ser considerada una herramienta, nunca un fin [en sí mismo]. (Weber 2012c, p. 126)

El criterio de la relación de valor se reduce en última instancia a la máxima de que las propiedades codificadas por el TI están siempre determinadas por el más amplio trasfondo teórico sobre el cual es construido. Este trasfondo aparta ciertos aspectos del fenómeno investigado — se le recuerda al lector el ejemplo de la I Guerra Mundial en la Sección 1-como relevante. Algunos TIS pueden resultar heurísticamente infructuosos, mientras que otros se sostendrán en la prueba y serán preservados como productivos con respecto a ciertos objetivos.

Como se mencionó antes, Weber vio los TIS como ocupados con la acción humana que, para él, era el tópico apropiado de las ciencias sociales. Todos los ejemplos de TIS que discute "representan" tipos de acción (por ejemplo, "acción instrumentalmente racional"), sistemas de creencias que motivan un tipo específico de acción ("el cristianismo"), tipos de agentes, grupos de agentes y sistemas que actúan, en los cuales la acción tiene lugar basada en motivos específicos ("organización", "feudalismo"), o tipos de procesos sociales

${ }^{15}$ Para ejemplos, véase Weiss (1981) sobre la recepción marxista de Weber. 
que resultan de la acción específicamente motivada ("el intercambio económico", "la racionalización"). Por lo tanto, al menos algunas de las propiedades codificadas por los TIS son intencionales: se ocupan de la razones, las finalidades o los valores que motivan la acción humana, y de las disposiciones a actuar sobre estos motivos de un modo específico. Un TI satisface el criterio de adecuación de significado si los motivos que codifica corresponden a las disposiciones que codifica.

Weber pensó que la evaluación de tal correspondencia depende de ciertas "reglas de experiencia" (Weber 2012a, p. 181) que están basadas en observación antecedente de la acción humana (Weber 1968, p. 10):

La interpretación de un curso coherente de conducta es "subjetivamente adecuada" (o "adecuada en el nivel del significado"), en tanto que, de acuerdo con nuestros modos habituales de pensamiento y sentimiento, sus partes componentes tomadas en su relación mutua son reconocidas como constituyendo un complejo "típico" de significado. (Weber 1968, p. 11)

Estas reglas de experiencia tienen el carácter de conocimiento del sentido común (Burger 1987: 86) que le permite uno juzgar un curso de acción como "entendible" dadas las circunstancias que lo motivan. Este aspecto de la concepción de Weber ha conducido a varias críticas: el "entendimiento" subjetivo difícilmente puede ser la base de una explicación científica de la realidad social y su explicación causal. Frecuentemente, se le atribuye a Weber una posición de acuerdo con la cual el método propio de la ciencia social es el "entendimiento" (Verstehen) subjetivo, en tanto que distinguido de la explicación causal. Pero éste no es el lugar para tratar en detalle con la cuestión de la Verstehen. ${ }^{16}$ Creo, sin embargo, que la exigencia de "inteligibilidad" no necesita ser vista como una apelación al entendimiento esotérico. ${ }^{17}$ También puede ser interpretado simplemente como significando que los medios (o cursos de acción) seleccionado para el TI deben corresponder a los fines (o motivos) seleccionados, sobre la base de un contexto más amplio de conocimiento empírico ("reglas del experiencia"), que puede ser de una naturaleza precientífica ("sentido común") o científica. En otras palabras: si un TI codifica propiedades concernientes a motivos y disposiciones a actuar, los motivos

\footnotetext{
${ }^{16}$ Pero véase Burguer (1987), de acuerdo con el cual Weber "se opuso radicalmente a cualesquiera de los tales argumentos que postulaban un método especial de entendimiento" (Burger 1987, p. 104).

${ }^{17}$ Después de todo, Weber mismo sugirió a sus contemporáneos que enfatizaban el papel de la intuición: "el que anhela ver (Schau) debiera ir al cine [...]" (Weber 1992, p. xli). Para las observaciones críticas de Weber sobre el psicologismo en las ciencias sociales, véase (Weber 1968, pp. 18-19).
} 
deben corresponder, de acuerdo con "reglas" preexistentes, a las disposiciones, y los motivos mismos no deben estar en conflicto. ${ }^{18}$ Esto se puede determinar mediante inferencia a partir de las reglas de experiencia en la medida en que éstas sean conocidas.

Weber balancea el criterio de adecuación de significado con el criterio de adecuación causal. La relación entre eventos, propiedades, etcétera, es causalmente relevante si "hay una probabilidad, que en el raro caso ideal raro pueda ser numéricamente establecida, pero que siempre es en algún sentido calculable, de que un evento observado dado (público o subjetivo) será seguido o acompañado por otro evento" (Weber 1968, pp. 11-12). En la construcción de un TI nosotros - en el "caso ideal"- dependemos no solamente del conocimiento de la relación causal, y por lo tanto "significativa", entre un motivo y una acción, sino también del conocimiento nomológico de su relación causal. Ambos criterios de adecuación deben estar, de acuerdo con Weber, unidos. ${ }^{19}$ Si el criterio de adecuación de significado no es satisfecho, entonces el investigador es dejado con una mera "probabilidad estadística incomprensible" (Weber 1968, p.12); si, por otra parte, falta la adecuación causal, entonces la supuesta relación entre motivos y cursos de acción tiene un carácter puramente hipotético (Weber 1968, pp. 9, 11) Weber no parece haber considerado la posibilidad de que el criterio de adecuación entrara en conflicto: que, por ejemplo, el criterio de significado lo guiara a uno a seleccionar motivos y disposiciones que se sabe que están totalmente desconectados estadísticamente. Sin embargo, a juzgar por el papel que le atribuye al conocimiento nomológico en las ciencias sociales, ${ }^{20}$ Probablemente habría visto tal conflicto como un obstáculo mayor en la construcción del tipo dado.

Para resumir, los objetivos cognitivos que guían los métodos abstracción e idealización en la construcción de TIS conducen a la selección de propiedades que

${ }^{18}$ Así es como interpreto la tesis de Weber de que el TI debiera ser un "cosmos internamente consistente de interrelaciones imaginadas" (Weber 2012c, p. 124).

19 "Una interpretación causal correcta de la acción típica significa que el proceso del cual se afirma que es típica muestra ser tanto adecuadamente aprehendido al nivel de significado como, al mismo tiempo, la interpretación es en algún grado causalmente adecuada" (Weber 1968, p. 12). De un modo similar, en el anterior "Objectivity": "Lo que nos ocupa es la construcción de relaciones que nuestra imaginación considera que está suficientemente motivadas y son por lo tanto 'objetivamente posibles', y que parecen adecuadas a la luz de nuestro conocimiento nomológico" (Weber 2012c, p. 126).

20 "Simplemente no es posible [... l llevar a cabo una imputación [causal] válida de algún efecto individual sin hacer uso de conocimiento "nomológico" - conocimiento de las regularidades de relaciones causales" (Weber 2012c, p. 118). 
(1) son relevantes con respecto a un sistema preexistente de conocimiento;

(2) son intencionales y se corresponden de acuerdo con conocimiento empírico sabido;

(3) ocurren juntos en enunciados nomológicos antecedentes.

Es así que el método de construcción de TIS es un caso espcial de la aplicación de métodos de abstracción e idealización. Su especificidad se halla en la naturaleza peculiar de los objetivos cognitivos que presupone, como se resumen en los tres puntos de arriba. Esta especificidad es una consecuencia de la naturaleza del tópico de las disciplinas que, de acuerdo con Weber, necesariamente construyen TIS.

\section{Los TIS como objetos ideales}

Usando las conclusiones previas, reconstruiré ahora casos particulares de TIS leyendo las caracterizaciones y definiciones de Weber de los TIS como explicaciones breves del proceso de su construcción. Quizá el ejemplo más simple, para el cual Weber también provee un análisis básico, es el del "sujeto económico" de la economía teórica citado anteriormente. De acuerdo con Weber, la meta de la ciencia económica es primeramente y antes que nada la de aprehender los fenómenos más elementales en la vida económica de un hombre "plenamente desarrollado" (Weber 1990, p. 29). Con ese propósito en mente, esta disciplina construye un TI de un sujeto económico que carece de cualesquiera motivos que no estén directamente relacionados con la satisfacción de necesidades materiales; al mismo tiempo, este sujeto está caracterizado por tres propiedades que ningún "hombre empírico" ejemplifica: “ $\alpha$ ) un entendimiento perfecto de la situación actual —omnisciencia económica, $\beta$ ) elección sin excepciones de los medios más apropiados para el fin dado - 'economización' perfecta, $\gamma$ ) pleno uso de las capacidades propias en los servicios de adquirir bienes — 'impulso incesante de adquisición" (Weber 1990, p. 30).

La construcción del TI del "hombre económico", resumido en las líneas anteriores, puede ser interpretada como un procedimiento que combina abstracción e idealización. Desde que el objeto input, el cual es una representación verídica del "hombre empírico" y codifica así propiedades realistas, es 
primeramente transformado en un abstractum que solamente codifica las propiedades relevantes del punto de vista de la economía (Weber no especifica ésta), seleccionadas sobre la base de objetivos cognitivos preexistentes. En segundo lugar, este abstractum es usado como un objeto input en el método de la idealización y transformado en un objeto ideal. Esto codifica las tres propiedades mencionadas arriba que no son ejemplificadas por ningún objeto espaciotemporal. La selección de estas propiedades está basada sobre los objetivos cognitivos antecedentes.

Otros ejemplos de TIS incluyen los cuatro tipos de acciones sociales distinguidos en (Weber 1968, pp. 24 ss). Acción instrumentalmente racional, racional en valores, afectiva y tradicional son "tipos" en "forma conceptualmente pura" a los cuales "la acción real se aproxima más o menos cercanamente o, en muchos de los casos más comunes, que constituyen sus elementos" (Weber 1968, p. 26). La introducción de todos estos tipos puede ser reconstruida como un procedimiento que combina abstracción e idealización. El comentario de Weber al segundo tipo es instructivo:

ejemplos de pura orientación racional en valores serían las acciones de personas que, sin considerar el costo posible para ellas mismas, actúan para poner en práctica sus convicciones de lo que les parece requerido por el deber, el honor, la prosecución de la belleza, un llamamiento religioso, la lealtad personal o la importancia de alguna "causa" sin importar en qué consista ésta. (Weber 1968, p. 25)

Es así que el TI de la acción racional en valores es un objeto ideal que codifica exclusivamente los motivos correspondientes a la lista de arriba ("valores") y la disposición a actuar siempre con tales motivos sin considerar las consecuencias.

En (Weber 1924), Weber discute el TI de una "polis democrática de ciudadanos", caracterizada del siguiente modo:

El servicio militar y los plenos derechos ciudadanos han sido emancipados de la propiedad de la tierra y existe una tendencia (la cual, desde luego, nunca sería verdaderamente realizada, ni siquiera en el tiempo la democracia Ática más radical, en el dominio de la calificación para la función pública) de permitir a cualquiera capaz de servir a la flota que ocupé una posición, esto es: todos los ciudadanos como tales sin consideración de diferencias en la propiedad. (Weber 1924, p. 40, la traducción es mía) 
La "polis democrática de ciudadanos" es un objeto ideal que codifica (a) propiedades ejemplificadas por algún objeto espaciotemporal (trivialmente: por ejemplo, ser una forma de organización social), (b) propiedades que no son ejemplificadas por ningún objeto tal. Es el resultado de transformar un objeto input abstracto que representa, por ejemplo, la democracia Ática. Nuevamente, la construcción puede ser vista como procediendo en dos pasos. Primeramente, se forma un abstractum que codifica solamente algunas de las propiedades del objeto input. En un segundo paso, este abstractum es transformado en un objeto ideal que codifica propiedades contra fácticas (esto es, las arriba mencionadas).

De manera interesante, Weber observa que se pueden usar TIS en la construcción de nuevas TIS. Por ejemplo, el TI de una "asociación con propósitos" (Zweckverein) depende de la TI de la "acción instrumentalmente racional" y le permitie a uno introducir los TIS de "órganos de la asociación", "activos con propósitos” y “aparato coercitivo” (Weber 2012d, p. 285).

\subsection{La función heurística de los TIS}

Hemos visto que la construcción de TIS combina métodos que, en términos de su estructura y naturaleza, no difieren en principio de aquellos usados en las ciencias naturales. En esta sección me enfocaré en los métodos usados en los que se usan TIS construidos. Aquí también mostraré que estos son métodos estándar no empíricos que no son específicos a las ciencias sociales. Como pone en claro el resumen de las concepciones de Weber en la Sección 1, Weber pensó que el papel primario de los TIS era el de herramientas heurísticas. Esta creencia frecuentemente adopta la forma de la tesis de que los Tis no son un fin en sí mismos, sino más bien un medio; los TIS no constituyen conocimiento en sí mismos, sino que son medios para adquirir conocimiento. ${ }^{21}$ Esto es muy consistente con la aproximación usual a los métodos de abstracción e idealización en la filosofía de la ciencia (empírica), donde son vistos como instrumentos subordinados a la meta omniabarcante de adquirir conocimiento (empírico) con la ayuda del estudio de objetos abstractos (no realístas).

Weber consideraba a los TIS herramientas que se usan en la formulación de hipótesis acerca de las causas (probables) de los fenómenos sociales. Tal uso de los TIS se describe como sigue:

${ }^{21}$ Véase, por ejemplo, (Weber 2012 ss., p. 225), (Weber 2012c, p. 126), (Weber 2012b, p. 332). 
Por ejemplo, un pánico en la bolsa de valores puede ser analizado de la manera más conveniente intentando determinar primeramente cual hubiera sido el curso de acción si no hubiese sido influenciado por los afectos irracionales; es entonces posible introducir los componentes irracionales, explicativos de las desviaciones observadas de este curso hipotético. (Weber 1968, p. 6)

El "pánico en la bolsa de valores" es el evento a ser explicado refiriéndolo a su causa, esto es, a lo que Weber llama la "imputación causal" (Weber 2012, p. 51) de este evento a algún otro evento. En este caso, el segundo evento son los "afectos irracionales" que influencian las acciones de los agentes en el mercado de valores. Tal imputación presupone la identificación de la causa relevante. De acuerdo con Weber, esta identificación procede a través de la comparación de la situación factual con una hipotética, en la que los agentes actúan de un modo instrumentalmente racional puro. Déjeme tratar de examinar este ejemplo con más detalle, aunque — debido a una cierta parsimonia del escrito de Weber - a costa de tener que hacer algunas conjeturas.

La explicación que propone Weber aquí es contrastante: "los eventos siguen el curso $e$ en vez del $e^{\prime}$ debido a la presencia de las condiciones antecedentes $c$ en vez de $c^{\prime \prime}$. ${ }^{22}$ Para formular tal explicación debe estar disponible un contraste - en este caso, el curso de eventos esperado en condiciones de acción racional puramente instrumental. El TI de un agente instrumentalmente racional no proporciona la respuesta por sí mismo. El uso heurístico de un TI en la formulación de una explicación consistirá, por lo tanto, en dos pasos. Llamo al primero "el estudio del TI" y al segundo "la explicación contrastante del TI".

En el primer paso, la meta es determinar el contraste: cómo debiera "comportarse" un TI en una situación más o menos específica. Los problemas cognitivos que pueden ser resueltos por el estudio de un TI son ilustrados por las siguientes preguntas: “¿que medios elegiría un agente instrumentalmente racional si tuviese los medios $M_{1}, M_{2}, \ldots, M_{n}$ a su disposición y persiguiese el fin $E_{1}$ ?”, “¿Qué pasos podría dar un líder carismático en una situación $S$ para maximizar su influencia?”, “¿Cuáles de los tipos de ética religiosa $R_{1}, \ldots, R_{m}$ corresponden mejor a la orientación capitalista de búsqueda de la ganancia?". ${ }^{23}$ Debido a que los TIS son objetos abstractos sin ningunos correlatos

${ }^{22}$ Sobre la explicación contrastante, véase (Lipton 1990). En el contexto de las ciencias sociales, véase (Ylikoski 2011).

${ }^{23}$ Todas las TIS son de (Weber 1968). 
espaciotemporales, estas preguntas no pueden ser respondidas por la investigación empírica.

El punto de partida de la construcción de un TI es, entre otras cosas, algún conocimiento nomológico, por ejemplo acerca de la disposición de los seres humanos a actuar en una situación $S_{1}$ y sus variantes $S_{2}, \ldots S_{o}$ en el modo $K_{1}$ o sus variantes $K_{2}, \ldots, K_{o}$. Basado en éste y otro conocimiento que constituye los objetivos cognitivos, uno transforma algún objeto input en el objeto output. El TI resultante, por ejemplo, el de un agente instrumentalmente racional $i$, solamente codifica los motivos seleccionados y disposiciones a actuar. Puede ser así determinado inequívocamente acerca de este agente que en una situación $S_{1}$ su curso de acción será $K_{1}$. El estudio adicional del TI tiene que ver con encontrar cómo actuaría $i$ en una situación modificada $S_{1}^{\prime}$. Esquemáticamente el estudio de los TIS adopta la forma:

$$
\begin{aligned}
& S_{1}(i) \rightarrow K_{1}(i) \\
& S_{1}^{\prime}(i) \rightarrow ?
\end{aligned}
$$

Aquí, basada en el conocimiento de una cierta constelación de una situación y una acción, uno pregunta cuál sería la acción en una situación modificada. Éste esquema corresponde el procedimiento empleado en experimentos de pensamiento. ${ }^{24} \mathrm{Si}$ esto habrá de ser un "experimento imaginario intuitivo" que emplea la imaginación y la inferencia no deductiva, o un "experimento teórico imaginario", depende del contexto más amplio de conocimiento que forma el trasfondo de la construcción y estudio del TI. ${ }^{25} \mathrm{Si}$ una teoría lo suficientemente rica está disponible, entonces la evaluación de cómo el agente actuaría en una situación modificada puede ser cuestión de inferencia deductiva a partir de la teoría y ciertas suposiciones auxiliares.

Regresemos a la bolsa de valores en pánico. El investigador ha establecido que en una situación $S$ (por ejemplo ante la emergencia de información acerca de una caída en los precios de los activos), el agente real ha decidido actuar de una manera $K$ (por ejemplo vendiendo los activos, lo cual conduce a una caída adicional en los precios de los activos). Primeramente, el investigador debe implantar el TI de un agente instrumentalmente racional en tal situación.

\footnotetext{
${ }^{24}$ Aquí me apoyo en (Picha 2011), una obra que se enfoca en la epistemología de los experimentos de pensamiento.

${ }^{25}$ Sobre la distinción entre los dos tipos de experimento (de pensamiento) imaginarios, véase (Hempel 1965).
} 
Esto se hace, como se ha mostrado arriba, mediante un experimento de pensamiento. Basado en el conocimiento de que en una situación genérica $T$ el agente instrumentalmente racional $i$ actuaría de la manera $L$, se infiere que en la situación de la bolsa de valores $S$ el agente actuaría de la manera $K^{\prime}$ :

$$
\begin{aligned}
T_{1}(i) & \rightarrow L_{1}(i) \\
S_{1}(i) & \rightarrow K^{\prime}(i)
\end{aligned}
$$

De esta manera se obtiene el contraste $S(i) \rightarrow K^{\prime}(i)$ con la situación en el mundo real $S(a) \rightarrow K(a)$. La meta es explicar qué propiedades (motivos, creencias, disposiciones a actuar, etcétera) conducen al agente del mundo real $a$ a actuar del modo $A$.

En esta coyuntura, resalta la función heurística del TI. Las propiedades $A_{1}^{\prime}, \ldots, A_{r}^{\prime}$ que están codificadas por el TI son conocidas de antemano. Las características de la acción típica ideal $K^{\prime}$ fueron clarificadas por el experimento de pensamiento. Se sabe que al menos algunas de las propiedades $A_{1}^{\prime}, \ldots, A_{r}^{\prime}$ Son "causalmente adecuadas"con respecto a la acción $K^{\prime}$. Los aspectos del curso de acción del mundo real $K$ son conocidos a partir de la evidencia empírica. El TI guía al investigador, entonces, a identificar propiedades $A_{1}, \ldots, A_{s}$ del agente $a$ del mundo real que no están codificadas por el TI y, por lo tanto, podrían haber sido responsables de los aspectos en los que el curso de acción del mundo real $K$ difiere del curso de acción típico ideal $K^{\prime}$. La identificación de estas propiedades, así como su confirmación en $a$, son tema de ulterior investigación, la cual puede requerir que el investigador reúna más evidencia empírica; el papel del TI se halla en indicar qué propiedades no podían haber sido responsables por el curso de acción bajo investigación. ${ }^{26}$ Supongamos que se puede confirmar que el agente $a$ difiere del agente $i$ solamente en que carece de la propiedad $A_{i}^{\prime}$ y tiene la propiedad $A_{1}$. Uno puede entonces inferir que fue esta propiedad la que causó la "desviación" de la acción del mundo real respecto de la típica ideal. Se puede formular una explicación de la forma:

(En la situación $S$ ) tuvo lugar el curso de acción $K$, en vez del $K^{\prime}$ porque el agente $a$ tenía la propiedad $A_{1}$ en vez de la $A_{1}^{\prime}$.

\footnotetext{
${ }^{26} \mathrm{El}$ TI también puede, en combinación con otro conocimiento, guiar al investigador a identificar propiedades que son conocidas como incompatibles con las propiedades codificadas por el TI. Sea lo que fuere, Weber enfatizó exactamente este papel heurístico "negativo" de los TIS: "entre más rigurosa y precisamente haya sido construido el tipo ideal, y así, entre más abstracto e irrealista en este sentido sea, más capaz será de desempeñar sus funciones al formular terminología, clasificaciones e hipótesis" (Weber 1968: 21, las cursivas son mías).
} 
En el caso del pánico de la bolsa de valores, $A_{1}$ sería el "afecto irracional" responsable de la "desviación" respecto de la acción instrumentalmente racional pura.

El procedimiento que conduce a la explicación sobre la base de la propiedad $A_{1}$ no difiere en principio del bien conocido método de la diferencia, uno de los "Cánones de Inducción de Mill": a partir de la presencia de una propiedad en un caso y su ausencia en otro, uno infiere que es la causa que se estaba buscando. Desde luego, en línea con la creencia de Weber de que los TIS debieran servir en la formulación de hipótesis, la explicación resultante debiera ser vista como hipotética; es decir, una que ha sido confrontada con otros casos de cursos de acción similares en situaciones similares.

Está claro que el uso heurístico de los TIS es un método complejo que incluye otros métodos no empíricos (los métodos del experimento de pensamiento, la inferencia y la explicación) y puede incluir la recolección de evidencia suplementaria mediante métodos empíricos. Sin pretender agotar el tópico, puede ser es esquemáticamente capturado así:

(1) Basado en conocimiento preexistente de la situación bajo investigación $S$ y del curso de acción $K$, la identificación un TI pertinente $i$.

(2) Mediante un experimento de pensamiento, la identificación del curso típico ideal de acción $K^{\prime}$ del agente $i$ en la situación $S$.

(3) La identificación del conjunto de propiedades $C$ en que difiere el curso de acción del mundo real $K$, del agente del mundo real $a$, del curso típico ideal de acción $K^{\prime}$ del agente $i$.

(4) La identificación del conjunto de propiedades $A$ del agente del mundo real $a$ en las que difiere del agente típico ideal $i$, y que podrían ser por lo tanto responsables de la presencia de propiedades de $C$ en el curso de acción $K^{\prime}$.

(5) La formulación de una explicación contrastante en la que el explanandum se refiera a las especificidades del curso de acción $K$ y el explanans se refiera a las propiedades de $A$, y en la cual el contraste es el curso de acción $K^{\prime}$ y las propiedades relevantes del agente $i$.

Debe notarse que "acción" se entiende aquí en un sentido amplio —incluye acciones por agentes individuales, pero también procesos sociales más am- 
plios, mientras que "agentes" incluye grupos. En la reconstrucción de otros casos particulares de uso de los TIS, probablemente sea necesaria una modificación de algunas de las formulaciones de arriba, dependiendo de la naturaleza de la situación bajo investigación y del TI utilizado. ${ }^{27}$

La explicación formulada en la instrucción 5 tiene un carácter hipotético. Si se sostiene en la faz de evidencia adicional, se confirmará la fertilidad heurística del TI seleccionado en la instrucción 1. Si no, esto significa que el TI seleccionado no puede ser usado productivamente en el caso dado, y ha de buscarse otro. Para Weber, esto no es una razón para descartar completamente el TI anterior: "esto no excluye la posibilidad de usar ese" TI "en cualquier otro caso" (Weber 2012e: 84). En esto vio él una característica adicional peculiar a las disciplinas científicas sociales. Una ley de la naturaleza que sólo se aplica con excepciones es, para Weber, insostenible; mientras que un TI que solamente muestra ser útil en un puñado de casos tiene un lugar seguro en la ciencia social. Dejando de lado las muchas dificultades de las leyes como enunciados "estrictamente universales", esta comparación no parece ser demasiado justa. Ni siquiera en las ciencias naturales es extraño el uso de una multiplicidad de modelos "locales" con limitaciones específicas (Weisberg 2007, pp. 645-6), o incluso modelos falsos pero simples, computables o predictivamente poderosos (Bokulich 2011).

\section{Conclusión}

La literatura relevante ofrece una constelación de caracterizaciones de TIS de Weber: son modelos, ${ }^{28}$ imágenes, hipótesis o definiciones (Hufnagel 1971: 223), pero también conceptos límite de sistemas teóricos. He propuesto que veamos el método típico ideal como una combinación de dos métodos: el de la construcción de TIS y el de su uso heurístico. El primero de estos tiene el carácter de un método no empírico cuya estructura y naturaleza no difiere en principio de las múltiples aplicaciones de la abstracción y la idealización conocidas en las ciencias naturales. El objeto resultantes de la aplicación de este método, es decir el TI particular, es específico solamente en el sentido de que se ocupa de la acción humana y sus motivos. Esta propiedad es una

\footnotetext{
${ }^{27}$ La secuencia de instrucciones arriba bosquejada podría ser utilizada, por ejemplo, para reconstruir el ejemplo de una "sociedad artesanal" brevemente discutida en la primera sección.

${ }^{28}$ Véase, por ejemplo, (Janoska-Bendl 1965, p. 55), (Burguer 1987, p. 164), (Lindbekk 1992, p. 290) y (Mommsen 1992, p. 131)
} 
consecuencia de la naturaleza del tópico y de las metas cognitivas de las ciencias sociales. $^{29}$

El uso del último método puede incluir la aplicación de métodos empíricos en la identificación de las propiedades en la instrucción 4. Sin embargo, aquí vale también que los procedimientos empleados en el uso heurístico de los TIS no difieren en principio de aquellos usados rutinariamente en las ciencias naturales. Desde luego, la reconstrucción metodológica bosquejada arriba no responde la pregunta de si las ciencias sociales debieran usar el método típico ideal, ni de si de hecho lo usan. Pero, en tanto que lo hacen, dependen de una combinación de métodos que también están disponibles a las ciencias naturales.

\section{Referencias}

Albert, H., (1967), "Probleme der Wissenschaftslehre in der Sozialforschung", en R. König1967, pp. 38-64.

Aronovitch, H., (2012), "Interpreting Weber's Ideal-Types", Philosophy of the Social Sciences, vol. 42, no. 3, pp. 356-369.

Bielik, L., Kosterec, M., Zouhar, M., (2014a), "Model metódy (1): Metóda a problém”, Filozofia, vol. 69, no. 2, pp. 105-118.

Bielik, L., Kosterec, M., Zouhar, M., (2014b), "Model metódy (2): Inštrukcia a imperatív", Filozofia, vol. 69, no. 3, pp. 197-211.

Bielik, L., Kosterec, M., Zouhar, M., (2014c), "Model metódy (3): Inštrukcia a metóda", Filozofia, vol. 69, no.8, pp. 637-652.

Bielik, L., Kosterec, M., Zouhar, M., (2014d), "Model metódy (4): Aplikácia a klasifikácia”, Filozofia, vol. 69, no. 9, pp. 737-751.

Bokulich, A., (2011), "How Scientific Models Can Explain”, Synthese, vol. 180, no.1, pp. 33-45.

Bruun, H. H., Whimster, S., (2012), Max Weber: Collected Methodological Writings, Routledge, Oxford.

Brzeziński, J., Klawiter, A., Kuipers, T., Łastowski, K., Paprzycka, K., Przybysz, P., (2007) The Courage of Doing Philosophy: Essays Presented to Leszek Nowak, Rodopi, Amsterdam.

Burger, T., (1987), Max Weber's Theory of Concept Formation: History, Laws, and Ideal Types, Duke University Press, Durham.

Demeulenaere, P., (2011), Analytical Sociology and Mechanisms, Cambridge University Press, Cambridge.

${ }^{29}$ En un artículo reciente, Hilliard Aronovitch arribó a conclusiones que son, en parte, similares a las mías: la principal diferencia entre los objetos abstractos de las ciencias naturales, como "vacío absoluto" o "superficie sin fricción" y los TIS consiste, dice, en que éstos tienen que ver con agentes humanos (Aronovitch 2012, p. 361). Sin embargo, esta diferencia no debiera de ser exagerada. Podríamos, de manera similar, yuxtaponer los objetos abstractos de la biología y la física. 
Halas, J., (2015), “Abstrakcia a idealizácia ako metódy spoločensko-humanitných disciplín”, Organon F, vol. 22, no. 1, pp. 71-89.

Hempel, C. G., (1960), Fundamentals of Concept Formation in Empirical Sciences, Chicago University Press, Chicago.

Hempel, C. G., Oppenheim, P., (1936), Der Typusbegriff im Lichte der Neuen Logik, A. W. Sijthoff's Uitgeversmaatschappij N. V., Leiden

Hempel, C. G., (1965), "Typological Methods in the Natural and the Social Sciences", en C.G. Hempel 1965, pp. 155 -171.

Hempel, C. G., (1965), Aspects of Scientific Explanation and Other Essays in Philosophy of Science, The Free Press, New York.

Hufnagel, G., (1971), Kritik als Beruf: Der kritische Gehalt im Werk Max Webers, Frankfurt am Main, Propyläen.

Janoska-Bendl, J., (1965), Methodologische Aspekte des Idealtypus. Max Weber und die Soziologie der Geschichte, Duncker \& Humblot, Berlin.

Jones, M. R., (2005), "Idealization and Abstraction: A Framework", en M.R. Jones \& N. Cartwright 2005, pp. 173-217.

Jones, M. R., Cartwright, N., Idealization XII. Correcting the Model, Rodopi, Amsterdam

Knowles, D.,Explanation and Its Limits, Cambridge University Press, Cambridge.

König, R., (1967), Handbuch der empirischen Sozialforschung, Ferdinand Enke, Stuttgart.

Lindbekk, T., (1992), “The Weberian Ideal-type: Development and Continuities”, Acta Sociologica, vol. 35, pp. 285-297.

Lipton, P., (1990), “Contrastive Explanation”, en D. Knowles 1990, pp. 247-266.

Mommsen, W. J., (1992), "Ideal Type and Pure Type: Two Variants of Max Weber's Ideal-Typical Method”, en W.J. Mommsen 1992, pp. 121-132.

Mommsen, W. J., (1992), The Political and Social Theory of Max Weber, University of Chicago Press, Chicago.

Nowak, L., (1980), The Structure of Idealization: Towards a Systematic Interpretation of the Marxian Idea of Science, Springer, Dordrecht.

Nowak, L., (2000), The Idealizational Approach to Science: A New Survey. Disponible en http://www.staff.amu.edu.pl/ epistemo/Nowak/approach.pdf

Nowakowa, I., (2007), "The Method of Ideal Types versus the Method of Idealization”, en J. Brzeziński, A. Klawiter, T.A.F. Kuipers, K. Łastowski, K. Paprzycka \& P. Przybysz 2007, pp. 159-165.

Picha, M., (2011), Kdyby chyby. Epistemologie myšlenkových experimentů, Nakladatelství Olomouc, Olomouc.

Weber, M., (1924) “Agrarverhältnisse im Altertum”, en M. Weber 1924, pp. 1-288.

Weber, M., (1924), “Gesammelte Aufsätze zur Sozial - und Wirtschaftsgeschicht”, J. C. B. Mohr, Tübingen.

Weber, M., (1968), Economy and Society: An Outline of Interpretive Sociology, University of California Press, Berkeley. 
Weber, M., (1990), Grundriss zu den Vorlesungen über allgemeine (,theoretische“) Nationalökonomie, J. C. B. Mohr, Tübingen.

Weber, M., (1992), The Protestant Ethic and the Spirit of Capitalism, Routledge, London.

Weber, M., (2012a), "Critical Studies in the Logic of the Cultural Sciences", en H.H. Bruun \& S. Whimster 2012, pp. 139-184.

Weber, M. (2012b), "The Meaning of "Value Freedom" in the Sociological and Economic Sciences", en H.H. Bruun \& S. Whimster 2012, pp. 304-334.

Weber, M., (2012c), "The "Objectivity" of Knowledge in Social Science and Social Policy", en H.H. Bruun \& S. Whimster 2012, pp. 100-138.

Weber, M., (2012d), "On Some Categories of Interpretive Sociology", en H.H. Bruun \& S. Whimster 2012, pp. 73-301.

Weber, M., (2012e), "Roscher and Knies and the Logical Problems of Historical Economics", en H.H. Bruun \& S. Whimster 2012, pp. 3-94.

Weber, M., (2012f), "R[udolf] Stammler's "Overcoming" of the Materialist Conception of History", en H.H. Bruun \& S. Whimster 2012, pp. 185-226.

Weber, M., (2012g), "The Theory of Marginal Utility and the "Fundamental Law of Psychophysics", en H.H. Bruun \& S. Whimster 2012, pp. 242-251.

Weisberg, M., (2007), "Three Kinds of Idealization", The Journal of Philosophy, vol.104, no.12, pp. 636-659.

Weiss, J., (1981), Das Werk Max Webers in der marxistischen Rezeption und Kritik, Westdeutscher Verlag, Opladen.

Ylikoski, P., (2011), "Social Mechanisms and Explanatory Relevance", en P. Demeulenaere 2011, pp. 154-172.

Zalta, E. N., (1988), Intensional Logic and the Metaphysics of Intentionality, MIT Press, Cambridge. 